Kyle Auger, OMS IV, Gregory Shedlock, OMS IV, Kasey Coutinho, OMS IV, Nicole E. Myers, DO, MS and Santiago Lorenzo*, PhD, MS, MS

\title{
Effects of osteopathic manipulative treatment and bio-electromagnetic energy regulation therapy on lower back pain
}

https://doi.org/10.1515/jom-2020-0132

Received May 26, 2020; accepted November 23, 2020; published online March 2, 2021

\section{Abstract}

Context: Lower back pain (LBP) is prevalent and is a leading contributor to disease burden worldwide. Osteopathic manipulative treatment (OMT) can alleviate alterations in the body that leads to musculoskeletal disorders such as LBP. Bio-electromagnetic Energy Regulation (BEMER; BEMER International AG), which has also been shown to relieve musculoskeletal pain, is a therapeutic modality that deploys a biorhythmically defined stimulus through a pulsed electromagnetic field (PEMF). Therefore, it is possible that combined OMT and BEMER therapy could reduce low back pain in adults more than the effect of either treatment modality alone.

Objectives: To investigate the individual and combined effects of OMT and BEMER therapy on LBP in adults.

Methods: Employees and students at a medical college were recruited to this study by email. Participants were included if they self-reported chronic LBP of 3 months' duration or longer; participants were excluded if they were experiencing acute LBP of 2 weeks' duration or less, were currently being treated for LBP, were pregnant, or had a known medical history of several conditions. Ultimately, 40 participants were randomly assigned to four treatment groups: an OMT only, BEMER only, OMT+BEMER, or control (light touch and sham). Treatments were given regularly over a 3 week period. Data on LBP and quality of life were gathered through the Visual Analog Scale (VAS), Short Form 12 item (SF-12) health survey, and Oswestry

\footnotetext{
*Corresponding author: Santiago Lorenzo, PhD, MS, MS, Lake Erie College of Osteopathic Medicine, 5000 Lakewood Ranch Blvd, Bradenton, FL, 34211-4909, USA, E-mail: slorenzo@lecom.edu Kyle Auger, OMS IV, Gregory Shedlock, OMS IV, Kasey Coutinho, OMS IV and Nicole E. Myers, DO, MS, Lake Erie College of Osteopathic Medicine, Bradenton, FL, USA
}

Low Back Pain Questionnaire/Oswestry Disability Index prior to treatment and immediately after the 3 week intervention protocol. One-way analysis of variance (ANOVA) was performed retrospectively and absolute changes for each participant were calculated. Normal distribution and equal variances were confirmed by Shapiro-Wilk test ( $>0.05$ ) and Brown-Forsythe, respectively. Significance was set at $\mathrm{p}<0.05$.

Results: Despite a lack of statistical significance between groups, subjective reports of pain reported on the VAS showed a substantial mean percentage decrease (50.8\%) from baseline in the OMT+BEMER group, compared with a $10.2 \%$ decrease in the OMT-only and 9.8\% in BEMER-only groups when comparing the difference in VAS ratings from preintervention to postintervention. Participants also reported in quality of life assessed on the Oswestry Low Back Pain Questionnaire/Oswestry Disability Index, with the OMT+BEMER group showing a decrease of $30.3 \%$ in score, the most among all groups. The OMT+BEMER group also reported the greatest improvement in score in the physical component of the SF-12, with an increase of $21.8 \%$. Conclusions: The initial data from this study shows a potential additive effect of combination therapy (OMT and BEMER) for management of LBP, though the results did not achieve statistical significance.

Keywords: BEMER therapy; lower back pain; musculoskeletal pain; OMT.

Low back pain (LBP) has a relatively high incidence and prevalence [1], affects people of all ages, and is a leading contributor to socioeconomic burden [2]. Chronic LBP is defined as occurring for 3 months or more, whereas acute LBP is defined as occurring for 2 weeks or less [3]. Individuals affected by LBP may experience progressive physical discomfort and may suffer psychological effects for several months; a proportion may remain severely disabled [3, 4]. The etiology of LBP is varied, from visceral causes to a lack of adequate blood flow to the muscles or 
musculoskeletal imbalance [5]. Inadequate blood supply and/or inadequate oxygen consumption can lead to fatigue and muscle pain [6-8]. For many people experiencing LBP, it is not possible to identify a specific nociceptive cause. Those suffering from LBP may recover but recurrence is common, and LBP often becomes persistent and disabling [9]. Despite the available management options, this back related disability and the number of individuals affected by it have increased steadily $[5,10]$. Recent changes to key recommendations in national clinical practice guidelines now emphasize self management, physical and psychological therapies, and some forms of complementary medicine (such as spinal manipulation, massage, or acupuncture), with less emphasis placed on pharmacological and surgical treatments [11].

Even with multiple clinical guidelines providing similar recommendations for managing LBP, a substantial gap between evidence and practice exists [12-15]. Osteopathic manipulative treatment (OMT) is a distinctive modality used by osteopathic physicians to complement conventional management of LBP. A previous randomized, controlled trial [14] of 455 patients found that OMT significantly improved patient outcomes and functionality compared with OMT sham treatment $(\mathrm{p}<0.001)$ and decreased the need for prescription analgesics $(\mathrm{p}=0.048)$. Franke et al. [16], in a meta-analysis of six studies ( $\mathrm{n}=769$ patients), reported clinically relevant effects of OMT for reducing pain (mean difference, $-14.93 ; 95 \%$ confidence interval $[\mathrm{CI}],-25.18$ to -4.68 ) and improving functional status (standard mean difference, -0.32 ; $95 \%$ CI, -0.58 to -0.07 ) in patients with low back pain. These studies paved the way for updated guidelines from the American Osteopathic Association in 2016, which stated, "The AOA believes that patients with low back pain should be treated with OMT given the high level of evidence that supports its efficacy.” [17]

An alternative approach for the treatment of LBP is the use of Bio-electromagnetic Energy Regulation (BEMER) therapy (BEMER International AG). BEMER is a therapeutic modality that deploys a biorhythmically defined stimulus through a pulsed electromagnetic field (PEMF). This stimulus has a targeted effect on the microvasculature, and the primary effect is an improvement in tissue microcirculation $[18,19]$. The positive effects of vasomotion on the microcirculation have been shown to result in significant increases in arteriovenous oxygen difference, the number of open capillaries, arteriolar and venular flow volume, and flow rate of red blood cells in the microvasculature [20, 21]. Several studies have reported promising outcomes in musculoskeletal pain management with the use of BEMER therapy [7, 22-24]. Furthermore, a systematic review [25] of six randomized, controlled trials investigating whether
PEMF was effective in low back pain showed that it resulted in a decrease in pain intensity and improved functionality for patients suffering from LBP. The reduction in pain intensity from baseline to the end point in that study ranged from 2.1 to 6.4 points out of 10 on the visual analog scale [25].

OMT has been shown to be effective in the treatment of LBP $[14,16]$. All osteopathic techniques use the concept of fascial connectivity throughout the body and help increase circulation and lymphatic flow [26, 27]. As discussed previously, BEMER therapy has been shown to increase microcirculation. Moreover, BEMER with physiotherapy showed reductions in pain and fatigue in patients with chronic low back pain [18]. The results from these studies suggest that a combination of OMT and BEMER therapy could potentially help increase circulation to myofascial structures that influence low back restriction and pain. The overarching goal of this study was to assess the individual and combined effects of OMT and BEMER therapy in patients with chronic LBP.

\section{Methods}

This study was approved by the Institutional Review Board at Lake Erie College of Osteopathic Medicine. Prior to the start of the study, written and informed consent was obtained from all research participants. The authors did not prospectively submit this study to a clinical trial registry, but it was registered post hoc at ClinicalTrials.gov (NCT04704375).

Subjects were compensated for their time with a $\$ 50$ gift card to a local grocery store.

\section{Study participants}

To investigate the individual and combined effects of OMT and BEMER therapy on LBP, participants were recruited and randomly placed into one of four treatment arms. A standardized recruitment email was sent to approximately 400 employees and students at Lake Erie College of Osteopathic Medicine. A total of 77 volunteers responded by emailing the lead student researcher, were recorded in an Excel spreadsheet, and were screened for eligibility by student researchers (K.A., G.S., K.C.) based on inclusion and exclusion criteria at the time when informed consent was obtained in hard copy. Participants were included if they self-reported chronic LBP 3 months or longer in duration [3]. Participants were excluded if they were experiencing acute LBP (2 weeks or less in duration), were currently undergoing treatment for LBP, were pregnant, or had a known medical history of 
psychiatric conditions, skin disorders, myositis, neurological symptoms in the lower extremities, cancer, bone fracture, deep vein thrombosis, osteopenia, a body mass index greater than 30 , or autoimmune disorder. Of the 77 respondents, 75 met inclusion criteria; two volunteers were excluded due to having acute LBP at the time of screening. Each of the 75 participants were assigned a unique number, and using the Microsoft Excel RAND function, a random number generator was used to select 40 participants to be involved in the study, because the authors wanted to establish four groups of 10 subjects each. Two participants dropped out during the first week due to scheduling conflicts. These two participants were replaced with participants from the original pool of screened volunteers (Figure 1). Random number generation using Microsoft Excel RAND and RANK functions was used to randomly place 10 participants in each of the four treatment groups: OMT only, BEMER only, OMT and BEMER (OMT+BEMER), or sham (light touch) therapy. The participants in each group were unaware of other treatment arms.

\section{Treatment groups}

The intervention protocol was 3 weeks long and the data collection ranged from February-September 2019, because the OMT and OMT+BEMER groups were treated between February and May, while the BEMER and control groups were treated between July and September. Participants in the OMT group received three treatments per week, participants in the BEMER group received five treatments per week, participants in the OMT+BEMER group received five BEMER and three OMT treatments per week, and participants in the control group received light touch and sham BEMER treatments at the same intervals. Participants who did not adhere to the full treatment regimen or experienced any adverse effects from treatment would have been removed from the study, but none were lost for those reasons. As noted earlier, two of the 40 participants were unable to complete the protocol and dropped out week one of treatment. They were replaced with two participants who had already been screened, met inclusion criteria, and signed informed consent. No adverse events were reported.

\section{Assessment and treatment protocols}

A standardized osteopathic assessment and treatment protocol was developed based on common dysfunctions associated with chronic low back pain [28]. This standardized assessment and treatment protocol was used to ensure consistency across participants, as all somatic dysfunctions could not be feasibly treated. This protocol was developed with a board certified NMM/OMM osteopathic physician (N.M.). The osteopathic structural exam and motion testing were focused on the lumbar spine, iliosacral, and sacral areas to diagnose dysfunctions commonly associated with chronic, nonspecific LBP [28]. The experience and comfort level of the osteopathic medical students and the supervising osteopathic physician (N.M.) were considered in the development of the treatment protocol. Common techniques that are well tolerated were identified and a treatment sequence was refined to reduce the number of patient position changes and operator variability.

Second year osteopathic medical students (G.S., K.A., K.C.) were trained for a minimum of $10 \mathrm{~h}$ and assessed on two separate occasions by a board certified NMM/OMM osteopathic physician (N.M.) to ensure uniform technique standardized protocol for each group. For every participant during each treatment session, a standardized osteopathic structural examination was performed, and diagnoses of somatic dysfunctions were made. If no

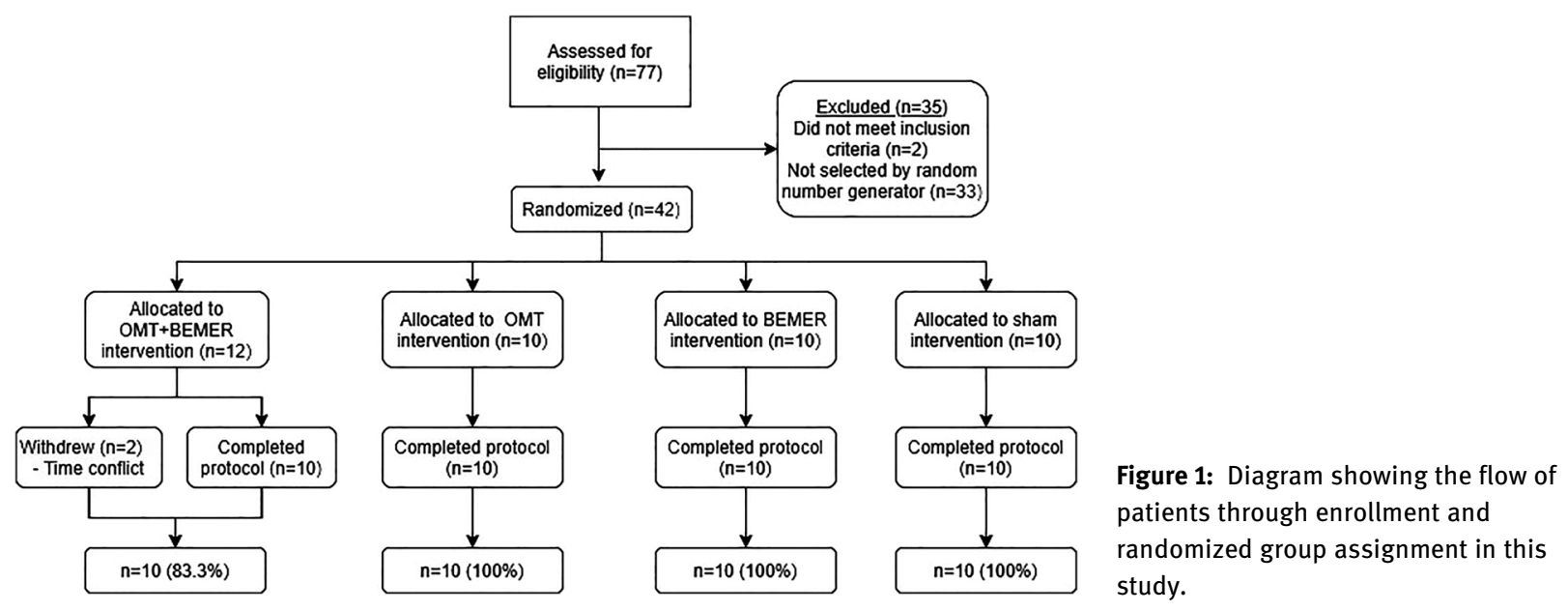


somatic dysfunctions were found in the areas examined, the associated part of the treatment protocol was not performed. The students performed the following protocol for each session and recorded the findings on a standard form:

- Observe and palpate thoracic and lumbar muscles for TART (tenderness, asymmetry, restriction of motion, and tissue texture) changes

- Perform lumbar intersegmental diagnosis

- Perform counterstrain tenderpoint assessment for psoas major, quadratus lumborum, and piriformis

- Perform spring test at the base of the sacrum

- Perform ASIS compression test to lateralize restriction in iliosacral mobility

After the osteopathic structural exam sequence was completed and recorded, participants receiving OMT were treated with a standardized sequence. Techniques were only applied to the areas where somatic dysfunction was found during the structural exam.

- Regional thoracic myofascial release (MFR), prone

- Lumbar soft tissue unilateral pressure, prone

- Psoas, piriformis, quadratus lumborum counterstrain, supine (only the most severe counterstrain tender point was treated)

- Lumbosacral/pelvic myofascial release, supine

- Sacrum balanced ligamentous tension, supine

- Lumbar muscle energy, seated

Participants receiving BEMER therapy laid supine on the BEMER mat (BEMER International AG) in a darkened and quiet room, with the B.Pad (BEMER International AG) on their lower back. The BEMER mat intensity setting was adjusted each week to progressively higher intensity. Intensity three was selected in week 1 , intensity four for week 2 , and intensity five for week 3. B.Pad ${ }^{\circledR}$ settings were set for Program 1 (8 min long) in week 1, Program 2 (16 min long) in week 2, and Program 3 (20 min long) in week 3. These settings were selected based on the manufacturer's recommendations. For the participants in the combination therapy group, OMT was performed prior to BEMER therapy on the 3 days the therapies overlapped.

Patients in the control group received light touch and BEMER sham treatments. The trained students placed their hands lightly on lumbar and sacral regions to mimic MFR techniques. However, no lifting or action was done. After the light touch treatment, the subject laid supine on the deactivated BEMER mat.

All treatment sessions, including the osteopathic structural exam, lasted no longer than $30 \mathrm{~min}$ for all groups.

\section{Outcomes assessments}

Prior to intervention and within 30 min after the 3 week intervention protocol, research participants were required to complete a paper version of validated surveys to assess pain and disability. The validated surveys used were a Visual Analog Scale (VAS) [29], the Short Form 12 item (SF-12) health survey (Appendix 1) [30], and the Oswestry Low Back Pain Questionnaire/Oswestry Disability Index (Appendix 2) [31]. SF-12 data were separated into a Physical Component Summary (PCS) and Mental Component Summary (MCS) [30]. The VAS score [29] measures the subjective pain along a $100 \mathrm{~mm}$ line continum from "no pain" to "pain as bad as it could possibly be." The SF-12 health survey [30] is a self-reported survey that measures the effects of health on daily activities. The Oswestry Low Back Pain Questionnaire [31] is a subjective tool to measure the functional effects of low back pain on everyday life that could lead to disability.

All outcomes analyses were performed in a blinded fashion with the subjects' survey and corresponding data deidentified and analyzed retrospectively; the survey contained only the participants' ID numbers. Absolute changes in questionnaire scores from preintervention to postintervention were calculated for each participant. A one-way analysis of variance (ANOVA) was used to determine any statistical significance between mean changes in the four groups. Normal distribution and equal variances were confirmed by Shapiro-Wilk test and Brown-Forsythe, respectively. Significance was set at $\mathrm{p}<0.05$, and values are presented as means \pm standard deviation (SD).

\section{Results}

The 40 research participants had a mean age of 25.1 years (SD, \pm 2.0 years); 21 (52.5\%) were women and 19 (47.5\%) were men. Demographics for the four treatment arms were: OMT only (25.9 \pm 2.5 years, four [ $40.0 \%]$ women), BEMER therapy only (24.6 \pm 1.6 years, five [50.0\%] women), OMT and BEMER (24.8 \pm 1.8 years, six [60.0\%] women), and sham therapy (control; $25.3 \pm 1.6$ years, six [60.0\%] women).

Preintervention mean scores for all groups are shown in the Table 1. One-way ANOVA analyses showed that preintervention mean values were not statistically different between groups.

All groups fell into the minimally disabled category for Oswestry score data, with an average score of 12.8 on the following scale: $0-4$ indicating no disability; 5-14 indicating minimal disability; 15-24 moderate disability; 25-34 severe disability; 35-50 complete disability [31]. PCS values 
Table 1: Preintervention scores.

\begin{tabular}{|c|c|c|c|c|c|c|c|c|}
\hline \multirow[t]{2}{*}{ Groups } & \multicolumn{2}{|c|}{ Visual analog scale } & \multicolumn{2}{|r|}{ Oswestry } & \multicolumn{2}{|c|}{ SF - 12 physical health } & \multicolumn{2}{|c|}{ SF - 12 mental health } \\
\hline & $\begin{array}{r}\text { Mean (SD) } \\
\text { Score }(0-100)\end{array}$ & $95 \% \mathrm{Cl}$ & $\begin{array}{r}\text { Mean (SD) } \\
\text { Score }(0-100)\end{array}$ & $95 \% \mathrm{Cl}$ & $\begin{array}{r}\text { Mean (SD) } \\
\text { Score }(0-100)\end{array}$ & $95 \% \mathrm{Cl}$ & $\begin{array}{r}\text { Mean (SD) } \\
\text { Score (0-100) }\end{array}$ & $95 \% \mathrm{Cl}$ \\
\hline Control $(n=10)$ & $34.1 \pm 26.0$ & $17.9-50.2$ & $10.8 \pm 9.0$ & $5.2-16.4$ & $48.6 \pm 6.9$ & $44.4-52.7$ & $54.1 \pm 4.6$ & $51.2-56.9$ \\
\hline OMT $(n=10)$ & $32.1 \pm 25.2$ & $16.5-47.7$ & $14.4 \pm 12.1$ & $6.9-21.9$ & $44.9 \pm 8.9$ & $39.4-50.4$ & $50.7 \pm 13.3$ & $42.4-58.9$ \\
\hline BEMER $(n=10)$ & $44.5 \pm 20.6$ & $31.7-57.3$ & $14.8 \pm 10.8$ & $8.1-21.5$ & $46.8 \pm 6.5$ & $42.8-50.8$ & $50.4 \pm 7.3$ & $45.7-54.9$ \\
\hline OMT + BEMER $(n=10)$ & $38.2 \pm 26.8$ & $21.6-54.8$ & $11.2 \pm 10.8$ & $4.5-17.9$ & $46.0 \pm 11.4$ & $38.9-53.1$ & $51.5 \pm 4.7$ & $48.6-54.4$ \\
\hline
\end{tabular}

$\mathrm{Cl}$, confidence interval; Oswestry, Oswestry Low Back Pain Questionnaire/Oswestry Disability Index; SD, standard deviation; SF-12, Short Form 12 item questionnaire.

on the SF-12 questionnaire were slightly below the national mean for all groups (mean, $46.6 \pm 8.4$; national mean, $50.0 \pm 10.0$ ), whereas the MSC values were slightly above the national mean (mean, $51.7 \pm 7.4$; national mean,
$50.0 \pm 10.0)$. These data suggest that our subjects had lower pain and disability compared with the national mean. The absolute change results for outcomes variable are shown in Figure 2A-D.
A

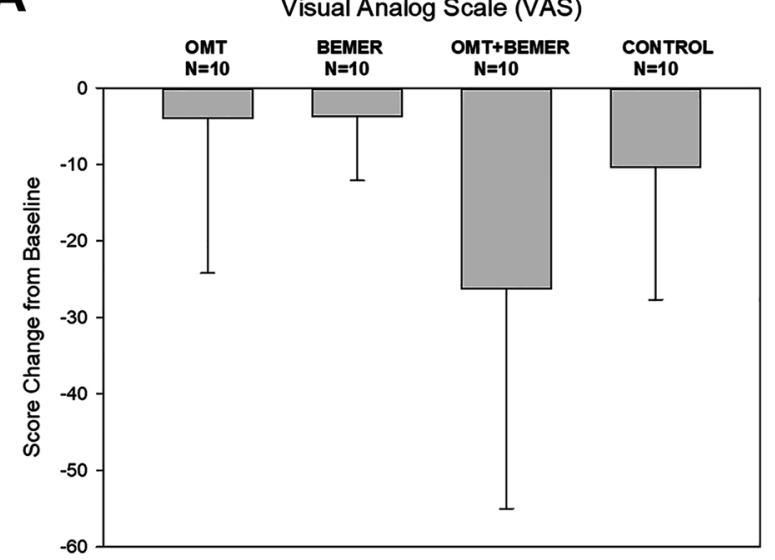

C

SF-12 Physical Component

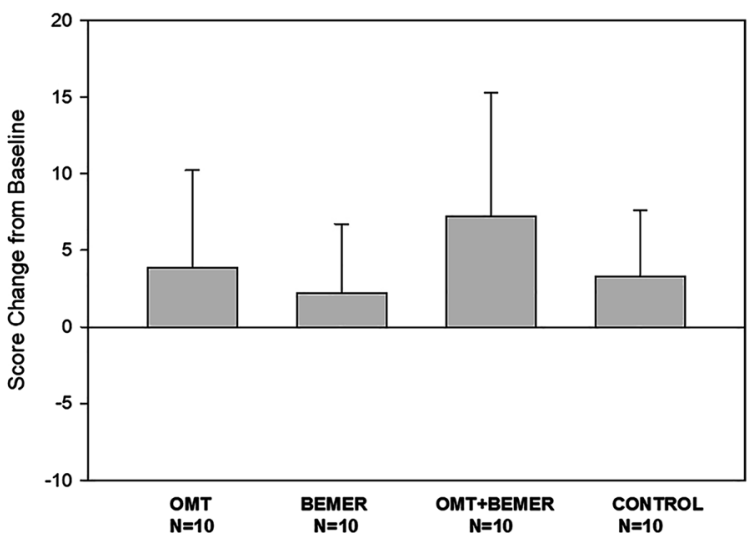

B

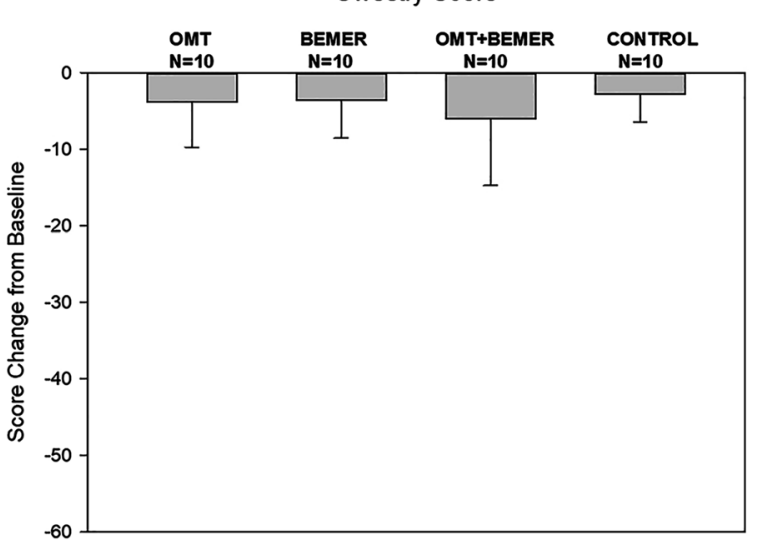

D

SF-12 Mental Component

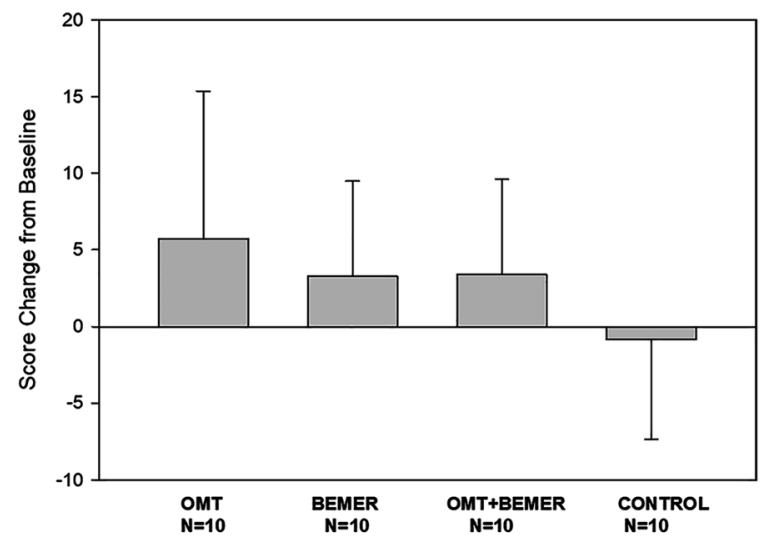

Figure 2: (A) Visual analog scale score changes from baseline. Values shown are absolute mean changes from preintervention to immediately after 3 week treatment intervention ( \pm standard deviation). There were no statistically significant differences between groups ( $p=0.06$ ).

(B) Oswestry Low Back Pain Questionnaire/Oswestry Disability Index score changes from baseline. Values shown are absolute mean changes from preintervention to immediately after 3 week treatment intervention ( \pm standard deviation). There were no statistically significant differences between groups $(p=0.68)$. (C) Short Form-12 (SF-12) questionnaire physical component score changes from baseline. Values shown are absolute mean changes from preintervention to immediately after 3 week treatment intervention ( \pm standard deviation). There were no statistically significant differences between groups $(p=0.29)$. (D) Short Form-12 (SF-12) questionnaire mental component score changes from baseline. Values shown are absolute mean changes from preintervention to immediately after 3 week treatment intervention ( \pm standard deviation). There were no statistically significant differences between groups $(p=0.26)$. 
For three of the four outcomes variables measured, the OMT+BEMER treatment group had decreased LBP and disability scores. For example, the OMT+BEMER group had a mean decrease of $26.2 \pm 28.8$ in their VAS score; the control group showed the next best decrease in the VAS with a $10.4 \pm 17.3$ mean decrease. The OMT group and the BEMER group were similar in result, with mean decreases of $3.9 \pm 20.3$ and $3.7 \pm 8.4$, respectively. The OMT+BEMER group had the largest change in mean Oswestry score, with a $6.0 \pm 8.7$ decrease, followed by the OMT group, with a $3.8 \pm 5.9$ decrease. The OMT+BEMER group also had the largest mean change in the physical component (PAS), with a $7.2 \pm 8.1$ mean increase. The next largest increase in score was from the OMT group, with a $3.9 \pm 6.3$ mean increase. The OMT group had the largest mean increase on the mental component of the SF-12 (MAS), with a $5.7 \pm 9.6$ increase, while the BEMER group and OMT+BEMER group followed with mean increases of $3.3 \pm 6.2$ and $3.4 \pm 6.2$, respectively.

One-way ANOVA analyses showed that absolute change values were not statistically different between groups $(\mathrm{p}>0.05)$.

\section{Discussion}

LBP is a major cause of disease burden and a major reason why patients seek medical attention [32]. Pain of any caliber can affect an individual's productivity and put them at risk for experiencing a decrease in quality of life. National guidelines for treatment of LBP have changed to focus on alternative therapies, including OMT, physiotherapy, and psychotherapy [11]. The overarching goal of the present study was to assess the individual and combined effects of OMT and BEMER therapy in adults with LBP. OMT as an adjunct to traditional treatment of LBP has been studied for cost effectiveness with promising results but more research is needed $[14,33,34]$. Osteopathic physicians who offer OMT also admit fewer patients to the hospital for LBP and have been shown to successfully treat LBP in fewer office visits [35]. Findings from our study could influence future studies and lead to incorporation of more OMT with BEMER therapy in clinical practice in the treatment of LBP, thus further decreasing healthcare costs and improving patient outcomes.

Our results suggest that the combination of OMT and BEMER therapy may have a positive impact on reducing LBP, although additional studies are warranted. Despite the lack of statistically significant differences between groups, the improvements in LBP observed in the OMT+BEMER group may be meaningful and clinically relevant; this could be demonstrated with a study of larger sample size and higher statistical power. Trends were observed showing that the combined OMT+BEMER treatment had a greater impact on LBP than each of the treatment modalities alone. The VAS analyses showed the largest decrease in LBP after 3 weeks of combined treatment (mean decrease, $26.2 \pm 28.8$ ). Moreover, the OMT+BEMER group showed the largest decrease in Oswestry score (mean decrease, $6.0 \pm 8.7$ ), lending evidence to the theory that combined treatment may lead to a more beneficial result than each treatment modality separately.

In a systematic review of six randomized controlled trials $(n=210)$ [25], PEMF therapy was shown to improve the functionality of individuals with LBP, but studies failed to show any added benefit when combined with standard therapy [25]. However, none of the studies in that review utilized OMT or BEMER Pro-Set as treatment modalities as ours did. Our results suggest there may be an additive effect between these two modalities. The observed decrease in LBP in our study could be due to the increase in microcirculation after OMT+BEMER sessions, bringing in new nutrients and flushing out cellular waste in a properly aligned lower back. Due to the low risk associated with these two therapies, BEMER can be considered a potentially promising adjunctive therapy in the treatment and management of LBP [14, 23]. Future studies should aim to adjust the protocol to maximize the beneficial effects of the combination treatment.

Gyulai et al. [18] investigated the synergistic effect of BEMER therapy with complex standard physiotherapy in 20 patients of 20-80 years of age with chronic LBP in a double-blind study. Results showed that in the shortterm, physiotherapy with BEMER therapy demonstrated a significant improvement in resting VAS (mean decrease of $26.8 \pm 13.7 ; \mathrm{p}=0.02$ ), but had no changes in the Oswestry scores and Quality of Life (determined by General Quality of Life Questionnaire SF 36; p>0.05). These results are comparable with our findings. Although our study did not achieve statistical significance, the change in the VAS scores observed in our study was remarkably similar to the results from Gyualai et al., which was approximately $26 \mathrm{~mm}$ on the $100 \mathrm{~mm}$ visual analogue scale [18].

The lack of statistical significance in our study could be attributed to several factors. First, our study groups consisted of only 10 subjects, whereas the Guyalai et al. study [18] included 20 subjects per group. Moreover, our subjects were young adults (mean age, 25.1 years), while their subjects were older adults (mean age, 67.3 years for men and 66.7 years for women) [18]. Finally, the baseline VAS scores were lower in our study, which leaves a smaller window for any potential improvements. Nevertheless, the results from our study and those from Gyulai et al. [18] suggest a possible potential benefit for the use of BEMER therapy in combination with OMT for the management and treatment of LBP. 
Our control group showed unexpected beneficial results, given that the research subjects in that group did not receive any actual treatments. This may have been one important reason for the lack of statistical significance observed, despite improvements in the treatment groups. That said, the subjects in the control group were put through diagnostic screening, went through light touch treatment, and had to lay in a quiet and darkened room for 20 min for the sham BEMER therapy. The sham BEMER therapy could have given the subjects a sense of relaxation, and the light touch of the researcher could have been inferred by the subject as treatment, leading to a placebo effect. It has been shown that simple touch can elicit a neural event, making pain more bearable [36].

\section{Limitations}

A small sample size was a limitation to our investigation. The low number of subjects per group $(\mathrm{n}=10)$ and the high variability of the measurements could have contributed to the nonsignificance of our results despite the postintervention improvements in some of our outcomes measures. Based on the present data, a power analysis estimated that 17 subjects per group would be needed to demonstrate significant differences between groups.

The length of the protocol must also be considered. Our study was designed to investigate the individual and combined effects of BEMER and OMT therapies. The BEMER protocol was adapted from the manufacturer's recommendations. To explore how OMT compared with or complemented BEMER therapy, we developed a shortened, more targeted OMT protocol. Future follow-up studies with longer OMT protocols are warranted. Furthermore, the low baseline values recorded preintervention would left a smaller window for any potential postintervention improvements. As shown in the preintervention measurements (Table 1), all groups were categorized in the minimally-disabled group by Oswestry score [31] and were generally below 40 out of 100 on the VAS score, placing them in the category of mild low back pain. Therefore, studies investigating the individual and combined effects of OMT and BEMER therapy in individuals with more severe LBP are warranted.

Another limitation that could have led to high variability in our results was the use of second year osteopathic students to perform the standardized treatment protocol. Although the students were trained on the protocol for a minimum of $10 \mathrm{~h}$ and assessed on multiple occasions, their limited clinical experience could have altered the effectiveness of treatment and ultimately affected the external validity of the study.

\section{Future studies}

There is a definite need for future studies to expand on the findings from our investigation. Additional studies with larger sample sizes to improve the statistical power and validity of the results are warranted. Also, future studies are needed to compare acute vs. chronic LBP and the potential benefits of OMT and/or BEMER treatment for each. The treatment protocol developed for this study could also be adapted for other musculoskeletal complaints such as hip and neck pain. Further research is needed regarding the effects of OMT and BEMER therapy on LBP, including standardized protocols, larger samples, and adjustment for low back pain confounders in order to achieve stronger conclusions.

\section{Conclusions}

This study investigated the individual and combined effects of OMT and BEMER therapy for LBP. Our results showed that a combination of OMT and BEMER therapy produced additive, although not statistically significant, effects on decreasing the level of LBP and increasing functionality in 40 volunteer participants. While more studies are warranted to further investigate the combined effects of OMT and BEMER therapy, the trends observed in our study shed light on the possibility of utilizing a combination of OMT and BEMER in the management of LBP in clinical practice.

Acknowledgments: The authors thank Kyle Herout, OMS IV, Thompson Fillmer, OMS IV, Melissa Damaske, OMS III, Nicholas Dominick, OMS III, Courtney Croley, OMS III, Blake Burch, OMS III, Luke McFarland, OMS III, Susan Tucker, OMS III and Daniel Danford, OMS III for their help with data collection.

Research funding: The current study was funded by an Internal Seed Grant from Lake Erie College of Osteopathic Medicine (grant No. 2018-19-25-154) for purchase of the BEMER equipment and compensation for participants' time.

Author contributions: All authors provided substantial contributions to conception and design, acquisition of data, or analysis and interpretation of data; all authors drafted the article or revised it critically for important intellectual content; all authors gave final approval of the version of the article to be published; and all authors agree to be accountable for all aspects of the work in ensuring that questions related to the accuracy or integrity of any part of the work are appropriately investigated and resolved.

Competing interests: None reported. 
Informed consent: Prior to the start of the study, written and informed consent was obtained from all research participants.

Ethical approval: This study was approved by the Institutional Review Board at Lake Erie College of Osteopathic Medicine. The authors did not prospectively submit this study to a clinical trial registry, but it was registered post hoc at ClinicalTrials.gov (NCT04704375).

\section{References}

1. Licciardone JC. The epidemiology and medical management of low back pain during ambulatory medical care visits in the United States. Osteopath Med Prim Care 2008;2:11.

2. Katz JN. Lumbar disc disorders and low-back pain: socioeconomic factors and consequences. J Bone Joint Surg Am 2006;88(Suppl 2): 21-4.

3. Koes BW, van Tulder MW, Thomas S. Diagnosis and treatment of low back pain. BMJ 2006;332:1430-4.

4. Manek NJ, MacGregor AJ. Epidemiology of back disorders: prevalence, risk factors, and prognosis. Curr Opin Rheumatol 2005;17:134-40.

5. Freburger JK, Holmes GM, Agans RP, Jackman AM, Darter JD, Wallace AS, et al. The rising prevalence of chronic low back pain. Arch Intern Med 2009;169:251-8.

6. Katayama K, Amann M, Pegelow DF, Jacques AJ, Dempsey JA. Effect of arterial oxygenation on quadriceps fatigability during isolated muscle exercise. Am J Physiol Regul Integr Comp Physiol 2007;292:R1279-86.

7. Haase R, Piatkowski J, Ziemssen T. Long-term effects of BioElectromagnetic-Energy Regulation therapy on fatigue in patients with multiple sclerosis. Altern Ther Health Med 2011;17:22-8.

8. Bohn W, Hess L, Burger R. The effects of the "physical BEMER ${ }^{\circledR}$ vascular therapy", a method for the physical stimulation of the vasomotion of precapillary microvessels in case of impaired microcirculation, on sleep, pain and quality of life of patients with different clinical pictures on the basis of three scientifically validated scales. J Complement Integr Med 2013;10(Suppl):S5-12, S5-13.

9. Hartvigsen J, Hancock MJ, Kongsted A, Louw Q, Ferreira ML, Genevay S, et al. What low back pain is and why we need to pay attention. Lancet 2018;391:2356-67.

10. Hoy D, March L, Brooks P, Woolf A, Blyth F, Vos T, et al. Measuring the global burden of low back pain. Best Pract Res Clin Rheumatol 2010;24:155-65.

11. Foster NE, Anema JR, Cherkin D, Chou R, Cohen SP, Gross DP, et al. Prevention and treatment of low back pain: evidence, challenges, and promising directions. Lancet 2018;391:2368-83.

12. Chou R, Qaseem A, Snow V, Casey D, Thomas Cross J Jr, Shekelle $P$, et al. Diagnosis and treatment of low back pain: a joint clinical practice guideline from the American College of Physicians and the American Pain Society. Ann Intern Med 2007;147:478-91.

13. Cronholm PF, Nicklin DE. Is spinal manipulation an effective treatment for low back pain? No: evidence shows no clinically significant benefit over watchful waiting. Am Fam Physician 2012; 85:763-4.

14. Licciardone JC, Minotti DE, Gatchel RJ, Kearns CM, Singh KP. Osteopathic manual treatment and ultrasound therapy for chronic low back pain: a randomized controlled trial. Ann Fam Med 2013;11:122-9.

15. Scott NA, Moga C, Harstall C. Managing low back pain in the primary care setting: the know-do gap. Pain Res Manag 2010;15: 392-400.

16. Franke H, Franke JD, Fryer G. Osteopathic manipulative treatment for nonspecific low back pain: a systematic review and metaanalysis. BMC Musculoskelet Disord 2014;15:286.

17. Task Force on the Low Back Pain Clinical Practice Guidelines. American osteopathic association guidelines for osteopathic manipulative treatment (OMT) for patients with low back pain. J Am Osteopath Assoc 2016;116:536-49.

18. Gyulai F, Raba K, Baranyai I, Berkes E, Bender T. BEMER therapy combined with physiotherapy in patients with musculoskeletal diseases: a randomised, controlled double blind follow-up pilot study. Evid Based Complement Alternat Med 2015;2015:245742.

19. Bernat SI. [Effectiveness of pentoxifylline and of bioelectromagnetic therapy in lower limb obliterative arterial disease]. Orv Hetil 2013;154:1674-9. A pentoxifillin es a bioelektromagnes-regulacios kezeles hatasa also vegtagi obliterativ veroerbetegsegben. Hungarian.

20. Klopp RC, Niemer W, Schmidt W. Effects of various physical treatment methods on arteriolar vasomotion and microhemodynamic functional characteristics in case of deficient regulation of organ blood flow. Results of a placebo-controlled, doubleblind study. J Complement Integr Med 2013;10(Suppl):S39-46, s41-9.

21. Klopp RC, Niemer W, Schulz J. Complementary-therapeutic stimulation of deficient autorhythmic arteriolar vasomotion by means of a biorhythmically physical stimulus on the microcirculation and the immune system in 50-year-old rehabilitation patients. J Complement Integr Med 2013;10(Suppl):S29-37, s31-9.

22. Piatkowski J, Kern S, Ziemssen T. Effect of BEMER magnetic field therapy on the level of fatigue in patients with multiple sclerosis: a randomized, double-blind controlled trial. J Altern Complement Med 2009;15:507-11.

23. Kanaparthi A, Kesary SPR, Pujita C, Gopalaiah H. Bio Electro Magnetic Energy Regulation (BEMER) therapy in myofascial pain dysfunction syndrome: a preliminary study. J Oral Biol Craniofac Res 2020;10:38-42.

24. Benedetti MG, Cavazzuti L, Mosca M, Fusaro I, Zati A. Bio-ElectroMagnetic-Energy-Regulation (BEMER) for the treatment of type I complex regional pain syndrome: a pilot study. Physiother Theory Pract 2020;36:498-506.

25. Andrade R, Duarte H, Pereira R, Lopes I, Pereira H, Rocha R, et al. Pulsed electromagnetic field therapy effectiveness in low back pain: a systematic review of randomized controlled trials. Porto Biomed J 2016;1:156-63.

26. Chikly BJ. Manual techniques addressing the lymphatic system: origins and development. J Am Osteopath Assoc 2005;105: 457-64.

27. Ettlinger $\mathrm{H}$, Willard FH. Anatomy and physiology of the lymphatic system. In: Seffinger M, editor. Foundations of osteopathic medicine: philosophy, science, clinical applications, and research, 4th ed. Wolters Kluwer; 2018:174-93 pp. chap. 8 .

28. Loveless B, Hruby RJ, Nevins NA. Osteopathic neuromusculoskeletal medicine. In: Seffinger M, editor. Foundations of osteopathic medicine: philosophy, science, clinical applications, and research, 4th ed. Wolters Kluwer; 2018:1031 p. chap. 41. 
29. McCormack HM, Horne DJ, Sheather S. Clinical applications of visual analogue scales: a critical review. Psychol Med 1988;18: 1007-19.

30. Ware J, Kosinski M, Keller SD. A 12-Item Short-Form Health Survey: construction of scales and preliminary tests of reliability and validity. Med Care 1996;34:220-33.

31. Fairbank JC, Pynsent PB. The Oswestry disability index. Spine 2000;25:2940-52. discussion 2952.

32. Maher C, Underwood M, Buchbinder R. Non-specific low back pain. Lancet 2017;389:736-47.

33. Steel A, Sundberg T, Reid R, Ward L, Bishop F, Leach M, et al. Corrigendum to "Osteopathic manipulative treatment: a systematic review and critical appraisal of comparative effectiveness and health economics research"
[Musculoskelet. Sci. Pract. 27 165-175]. Musculoskelet Sci Pract 2017;30:86.

34. Verhaeghe N, Schepers J, van Dun P, Annemans L. Osteopathic care for low back pain and neck pain: a cost-utility analysis. Complement Ther Med 2018;40:207-13.

35. Reilly BM, Hart A, Evans AT. Part II. Evidence-based medicine: a passing fancy or the future of primary care? Dis Mon 1998;44: 370-99.

36. Benedetti F. Placebo and the new physiology of the doctorpatient relationship. Physiol Rev 2013;93:1207-46.

Supplementary Material: The online version of this article offers supplementary material (https://doi.org/10.1515/jom-2020-0132). 